\title{
Estimation as an Essential Skill in Entrepreneurial Thinking
}

\section{Dr. Daniel Raviv, Florida Atlantic University}

Dr. Raviv is a Professor of Computer \& Electrical Engineering and Computer Science at Florida Atlantic University. In December 2009 he was named Assistant Provost for Innovation and Entrepreneurship.

With more than 25 years of combined experience in the high-tech industry, government and academia Dr. Raviv developed fundamentally different approaches to "out-of-the-box" thinking and a breakthrough methodology known as "Eight Keys to Innovation." He has been sharing his contributions with professionals in businesses, academia and institutes nationally and internationally. Most recently he was a visiting professor at the University of Maryland (at Mtech, Maryland Technology Enterprise Institute) and at Johns Hopkins University (at the Center for Leadership Education) where he researched and delivered processes for creative \& innovative problem solving.

For his unique contributions he received the prestigious Distinguished Teacher of the Year Award, the Faculty Talon Award, the University Researcher of the Year AEA Abacus Award, and the President's Leadership Award. Dr. Raviv has published in the areas of vision-based driverless cars, green innovation, and innovative thinking. He is a co-holder of a Guinness World Record. His new book is titled: "Everyone Loves Speed Bumps, Don't You? A Guide to Innovative Thinking."

Dr. Daniel Raviv received his Ph.D. degree from Case Western Reserve University in 1987 and M.Sc. and B.Sc. degrees from the Technion, Israel Institute of Technology in 1982 and 1980, respectively.

Miss Alyssa J. Harris, Florida Atlantic University 


\title{
Estimation as an Essential Skill in Entrepreneurial Thinking
}

\author{
Daniel Raviv, Alyssa Harris, and Thiago Dezotti \\ Department of Computer \& Electrical Engineering and Computer Science \\ Florida Atlantic University \\ Boca Raton, FL 33431 \\ Emails: ravivd@fau.edu alyssaharris2013@fau.edu tcdezotti@gmail.com
}

\begin{abstract}
With technological advances, newer generations of students are exposed to computational tools that lead to more accurate results, and the old-fashioned 'art of estimation' is often being overlooked. While in many cases accuracy is imperative, there are frequent instances when 'back of envelope calculations' or rough estimation will suffice. Oftentimes, after calculations are made with the assistance of technology, students fail to question if their computations or simulation results actually make sense. This lost art of estimation can lead to precise calculations that are completely irrelevant. In engineering this could lead to design flaws and unsafe outcomes.
\end{abstract}

The goal of this paper is to briefly re-introduce the art of estimation in engineering. This is evident when dealing with entrepreneurial thinking where projections and estimations need to be quickly calculated and frequently modified as necessary. Estimation specifically allows entrepreneurs to make quick inexpensive decisions, which would otherwise cost time and resources. This paper shares research work that involves various, example-based estimation methods that are useful in engineering. The methods provide rough predictions of expected outcome, allowing students to intelligently guess a reasonable range of expected outcomes, given basic raw data within its necessary parameters. The methods include:

- Segmentation

- Fermi Estimation

- Rules of Thumb

- The 80/20 Rule

- Visual Method

- Upper and Lower Limit Estimations

For each method the authors introduce a brief history, a short explanation, as well as suggest how and when to use each method. Also included are classical examples and practice problems to reinforce students' knowledge.

In order to assess the validity of the proposed approach, a recent presentation about estimation methods followed by a detailed questionnaire was presented to students in a "Fundamentals of Engineering" class that comprised of freshman students resulting in 63 responses that are very favorable. Summary of results on a scale of " 1 " to " 5 ", "5" being strongly agree, "3" neutral, and " 1 " strongly disagree. The overall average response was 4.13 . This indicates that students preferred learning the presented estimations methods using an innovative and visual 
approach. Based on the additional questions, it turns out that students prefer to be taught, to learn visually and intuitively, to use PowerPoint and instructor notes and not rely on teaching themselves.

\section{Introduction}

Estimation, the act of judging the size, speed, amount, or cost of something, is a valuable method that can be used to make important predictions and decisions ${ }^{1}$. Estimation has become a subconscious skill that we all use on a daily basis, whether it be determining if you have time to pick up a coffee on the way to work, or if you need those few minutes to prepare for your presentation that morning. Generally, our predictions are "on point" because we can draw on our past experiences. If asked by a visitor how long would it take to get to the airport, you can reply with a quick estimate based on prior experience, the day of the week, time of the day, known road and traffic situation, and weather conditions. "Between 30 to 50 minutes" might be your reply. This answer is "good enough" for the person that asked the question. He/she does not need to know the exact time, but rather a "ball park" answer.

Even Engineers that are well-trained in Math and Science rely on estimation skills to make important decisions without getting caught up in the frequently time-consuming, complicated mathematical calculations ${ }^{2}$. Engineering students often have a difficult time navigating problems with tough calculations. By the time a student finds the solution of a problem, he/she simply moves on, satisfied with his/her work. No care is taken into account when a student, solving a purely resistive circuit, writes " $\mathrm{R}=-150 \Omega$ " as his/her final answer. However, disregarding any math equations, it is known that a resistor cannot have a negative value. Bourn and Baxter recognize that, "open-ended problems are challenging for many students because they often have little sense of what a "correct" answer would be and struggle with evaluating the quality of an answer derived from a calculator or computer model"3. Estimation in early stage of problem solving can help students determine what roughly to expect, i.e., whether or not their later detailed solution makes sense. Nachtmann and Lehrman advocate that "one of the greatest challenges" is educating students on how to manage uncertainty and estimation ${ }^{4}$. Thus, it is pertinent to introduce students to the "art of estimation," that their heavy math and science curriculum does not emphasize ${ }^{1}$. This is especially relevant to students considering an entrepreneurship path because estimation enables you to have a rough idea of what you need to manage. Estimation helps make common sense decisions in a short period of time without utilizing excessive time or resources.

In this paper, the reader will be exposed to several well-known estimation methods that engineering students can benefit from. We are working on a manuscript that incorporates more engineering and entrepreneurship related methods, and more examples. This paper reports on work in progress, and is by no means comprehensive. Here, we specifically focus on Segmentation, Fermi, Rule of Thumb, 80/20 Rule, Visual estimation methods, and Upper Lower Limits. Further understanding of estimation will help students understand that this "lost art of estimation" can be a vital tool at their disposal. It is possible to exert less effort, and reach adequate results and conclusions using "back of envelop" calculations. It may be sufficient, especially in entrepreneurial thinking, where estimation of the time it takes to get a prototype, 
first customers, potential market, cost, etc. are crucial.

\section{Estimation Methods}

Our investigation began with a class led experiment to test whether students are responsive when introduced to the "art of estimation," using visual and engaging material. Students were introduced to multiple estimation methods designed to sharpen their estimation skills. As a result, students were expected to utilize these methods to solve simple problems without the use of more complex, resource-intense calculations.

Students were exposed to six techniques of estimation, including Segmentation, the Fermi method, Rules of Thumb, the 80/20 Rule, Upper and Lower Limits, and the Visual method. They were then asked to complete a questionnaire based on their immediate understanding of the methods. At a later point, students were asked successive questions on their preferences and overall understanding of the methods to assess whether the given teaching method was effective.

\section{Segmentation}

The first approach students were introduced to, or rather re-introduced to, was the Segmentation Method. The Segmentation Method is synonymous with the classic elementary school question, "How many beans are in this jar?", This can seem like a daunting task, if your procedure is to count each bean.

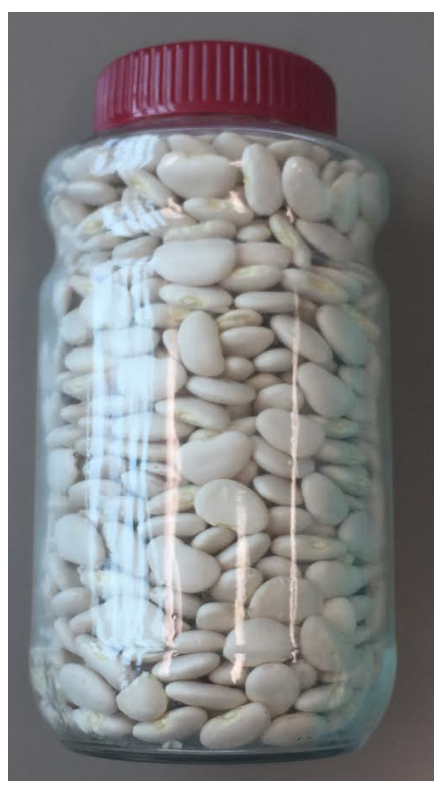

Figure 1: How many beans are in this jar?

Luckily, the Segmentation Method can break down time-consuming tasks by following a simple procedure ${ }^{6}$ :

1. Break up (or segment) larger quantities into smaller quantities

2. Count the smaller quantity 
3. Estimate how many of the smaller quantities make up the larger quantity

In terms of beans:

If you know how many beans can fit into a small jar, say 60 beans, and estimate how many small jars can fit in a large jar, say 15, you end up with a guess of 900 beans, which is close to that of the actual number of 850 beans (which we actually counted) ${ }^{7}$.

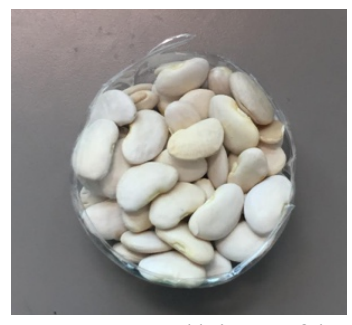

Figure 2: Small jar of beans

One of the benefits of estimation is that the actual number of beans is not necessarily relevant. If this jar were set up in an elementary school classroom, the child with the closest guess is the winner. (In case of a competition, it would be nice to also have the actual number as a reference... just to avoid awarding the "wrong" winner.)

Although, engineers can not necessarily "win" in the real-world by estimating to the closest value, they can save time and effort by getting close. Segmentation can allow engineers to break problems into manageable pieces. Engineers and Entrepreneurs set out to make what was previously impossible possible. In order to accomplish such a feat, segmentation can be utilized to break the problem into manageable pieces. If you know something about a smaller group, it can be applied to a larger group, and suddenly the problem is no longer overwhelming.

\section{Fermi Estimation}

Next, students were introduced to the Fermi Method, that enables them to make roughly-accurate estimates with very little data. Although this type of estimation yields an answer that is likely to be off by a factor of 2 or more, getting an estimate within a factor of 5 is enough to make a decision in many instances.

The Fermi Method commences by collecting benchmarks related to the problem. A benchmark is a readily available or known fact. Once all of the benchmarks have been found, the second step is to use dimensional analysis, or unit analysis, to find a path to the answer. The third rule is to use worst-case scenario limits. In some cases, it can be difficult to guess a reasonable value, so by placing upper and lower bounds, or limits, although the answer is not precise, we can determine what the answer is not! With that, we can use other information until we can find a reasonable value to start the process of estimation. It is important to search for related information that can be obtained on the internet to increase accuracy. There is a section dedicated to upper and lower limits further on in the paper.

Now, imagine you are asked, "how many apples are on the tree?" 


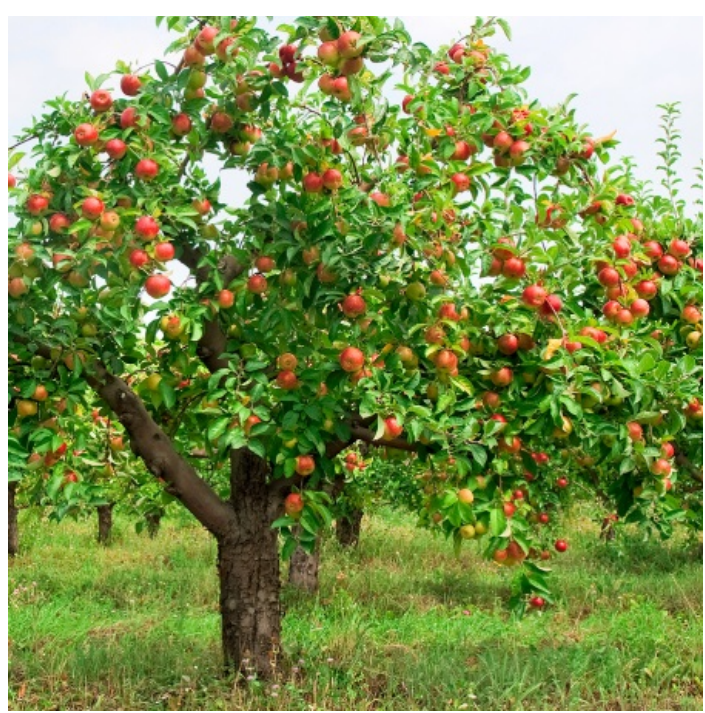

Figure 3: How many apples are on the tree? (http://www.albanhillnurseries.co.uk/alban-hillnurseries-feature-trees-bedford-residents-adore)

To figure out how many apples are on a tree, the Fermi Method tells us that you can use the estimated number of apples per branch, and estimated number of branches per tree to figure it out $^{8}$. Units cancel and the result is an answer in apples per tree.

Computation:

$\frac{12 \text { apples }}{1 \text { branch }} \times \frac{8 \text { branches }}{1 \text { tree }}=\frac{96 \text { apples }}{1 \text { tree }}$

Similarly, the Fermi Method can be used to calculate more abstract values. One of such estimations is the circumference of the earth by asking and answering questions we are familiar with ${ }^{9}$.

Benchmarks:

1. How many time zones do you pass through when you fly from New York to Los Angeles?

3

2. How many miles is it, about, over that same distance?

About 3000 miles

3. How many miles per time zone, on average?

About 1000 miles

4. How many time zones must there be around the world?

24 time zones as there are 24 hours in a day

5. How many miles around the world?

$$
\frac{1000 \text { miles }}{1 \text { time zone }} \times \frac{24 \text { time zones }}{1 \text { world }}=24000 \text { miles } / \text { world }
$$


The Fermi method can also be used in estimating the number of beans in a jar. Using the Fermi Method, the following questions can be asked: What is the approximate size of a bean? What shape are the beans? What is volume of a single bean? Do the beans completely fill the bottle? Then we look at the relevant background information. A one-liter bottle has a volume of 1000 $\mathrm{cm}^{3}$ and beans are shaped like a small cylinder that measures about $1.5 \mathrm{~cm}$ high, with a diameter of $1 \mathrm{~cm}$. The formula to calculate the volume of a cylinder is:

$$
V=\pi r^{2} h \text { (to keep things simple, use pi=3) }
$$

Now we can use all the information to try and win the prize by making an intelligent guess:

Since the beans are semi cylindrical, they won't be tightly packed in the bottle, approximately $80 \%$ of the volume of the bottle will be occupied. We can divide the occupied volume of the bottle by the volume of a single bean, to find out how many jelly beans are in the bottle.

Volume of one jelly bean $=1.5 \mathrm{~cm} \times 3 \times \frac{1}{2} \mathrm{~cm} \times \frac{1}{2} \mathrm{~cm}=1.125 \mathrm{~cm}^{3}$

Occupied volume of bottle $0.90 \times 1000 \mathrm{~cm}^{3}=900 \mathrm{~cm}^{3}$

$\frac{\text { Occupied volume of bottle }}{\text { Volume of each jelly bean }}=\frac{900 \mathrm{~cm}^{3}}{1.125 \mathrm{~cm}^{3}}=800$ beans

So we can estimate that there are 800 beans in the bottle. This method is sure to win the prize because when the beans were actually counted, there were 850. Amazingly, in this case, the Fermi Method yielded an answer that was very close to the actual number.

Now, imagine that you are the engineer working/ inspecting/consulting for a plant. You have now successfully identified the size of each object, and capacity of the tank with little work. However, you have also set yourself up to answer even more questions. How long will removal or the objects take? If you know the diameter of the drain, size of the objects and amount of objects, you can make a good guesstimate very quickly. In the case of emergency, when you are pressed for time, the Fermi Method will speed up your response ${ }^{10}$.

Another benefit of the Fermi Method, is that it considers units when making an estimation. Far too often students arrive at an answer that is off by a factor of the units. They multiply and divide correctly, but do not pay attention to their units in the process. Unfortunately, this problem has followed a few students into their careers. Major disasters, such as the NASA Orbiter and Gimli Glider are a result of this. In the case of the Gimli Glider, an Air Canada aircraft, the fuel was weighed and added in kilograms, rather than imperial units $(1 \mathrm{~kg} \neq 1 \mathrm{lb})^{11}$. However, Fermi accounts for this because your units must cancel in order to achieve your final answer. Fermi can also be used as an inspection to make sure that your calculated value is in the correct range or magnitude.

Unlike the Fermi Method, the next methods are essentially "facts of life" that most people experience. Rules of Thumb are used for specific estimations based on past experiences. For example, it is possibly referring to a rough measurement of an inch, since the first joint of an adult's thumb, is approximately one inch. Carpenters, who may have used the width of their 
thumbs to measure, before they had tape measures, could rely on this imprecise yet reliable, convenient measurement. This seems like a nice explanation, however, there is no definite documentation confirming this. Modernly, the expression has come to mean, a broad approximation using some basic information.

\section{Rules of Thumb}

Rules of Thumb can best be described as "a broadly accurate guide based on practice instead of theory". Tom Parker, a Rules of Thumb collector believes that their ingenuity stems from the fact that they are like, "a homemade recipe for making a guess, a mental tool that takes information you already have, and turns it into all the information you need"12. Unlike other estimations methods, Rules or Thumb are collective in nature, and tend to occur frequently in everyday life. It falls somewhere between a mathematical procedure and total speculation. It is a mental tool to help quickly assess a given situation and solve a problem. Some Rules of Thumb, are quantitative and others are more qualitative.

Imagine you have $\$ 100$ in the bank. Assuming compound interest how long will it take for you to double your money if the compound interest rate is $8 \%$ ?

Instead of grabbing your calculator and crunching the numbers, there is a rule of thumb for exponential growth at a constant rate, called the "Rule of 72 " for rates of growth (works best for interest rates ranging from $4 \%$ to $12 \%$ ). If you take the number 72 and divide it by interest rate, you arrive at a fairly accurate estimate with less work compared to the traditional method. Computation:

Rule of $72: \frac{72}{8}=9$ YEARS

It takes 9 years @ 8\% compound interest to double your $\$ 100$

Traditional method of calculating interest: $A=P\left(1+\frac{R}{100}\right)^{N}$, where $\mathrm{A}=$ final value, $\mathrm{P}=$ principle, $\mathrm{R}=$ rate, $\mathrm{N}=$ number of years.

This rule is precise, but is most precise when you stay in the interest rate range of $7-9 \%$ as can be seen in Figure 4. 


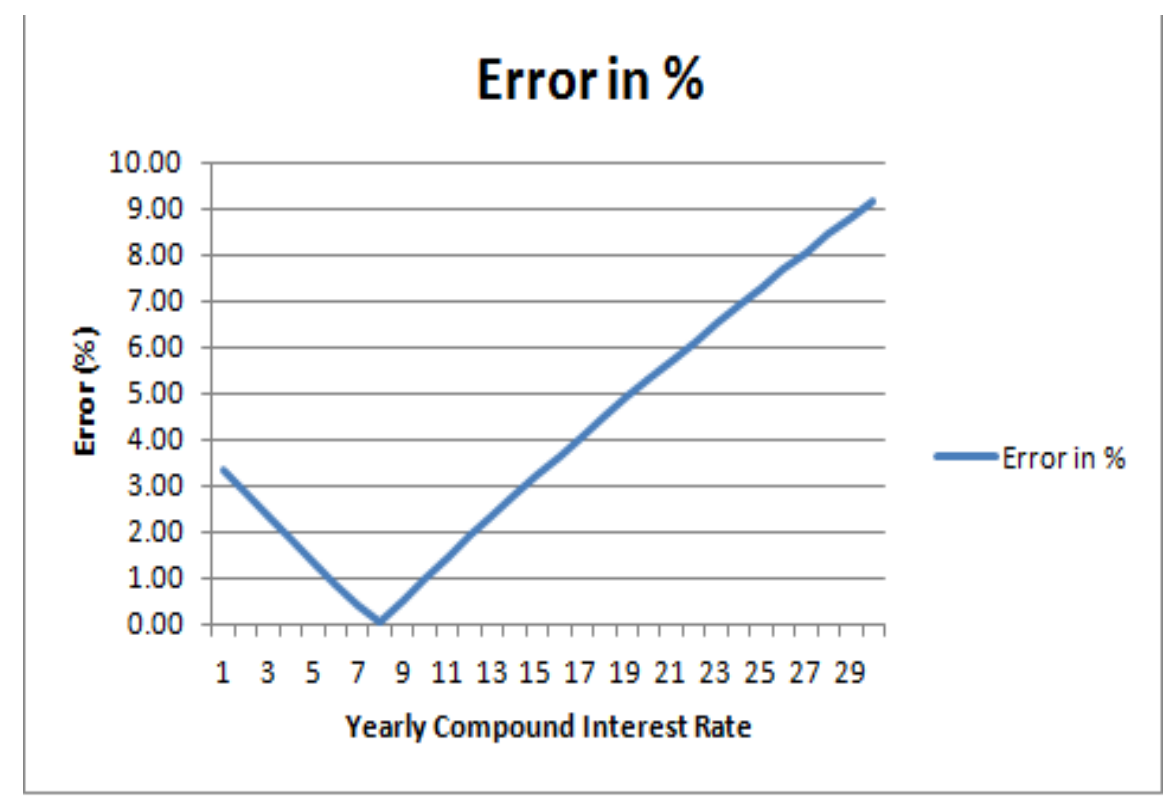

Figure 4: Rule of 72 Error Percentage Plot

Some Rules of Thumb that engineering students may be familiar with are:

a. For every hour you spend in class, you need to spend two hours studying.

b. Moore's Law on Technology: "The number of transistors in a dense integrated circuit doubles approximately every 2 years."

.... And the list goes on.

\section{0/20 Rule}

Have you ever noticed that the majority of the work gets accomplished by a small group of people? Another well-known Rule of Thumb is the $80 / 20$ Rule which states that $80 \%$ of outcomes can be attributed to $20 \%$ of the causes for a given event. That is, $80 \%$ of the work gets done by only 2 out of the 10 people, as in Figure 5 . 


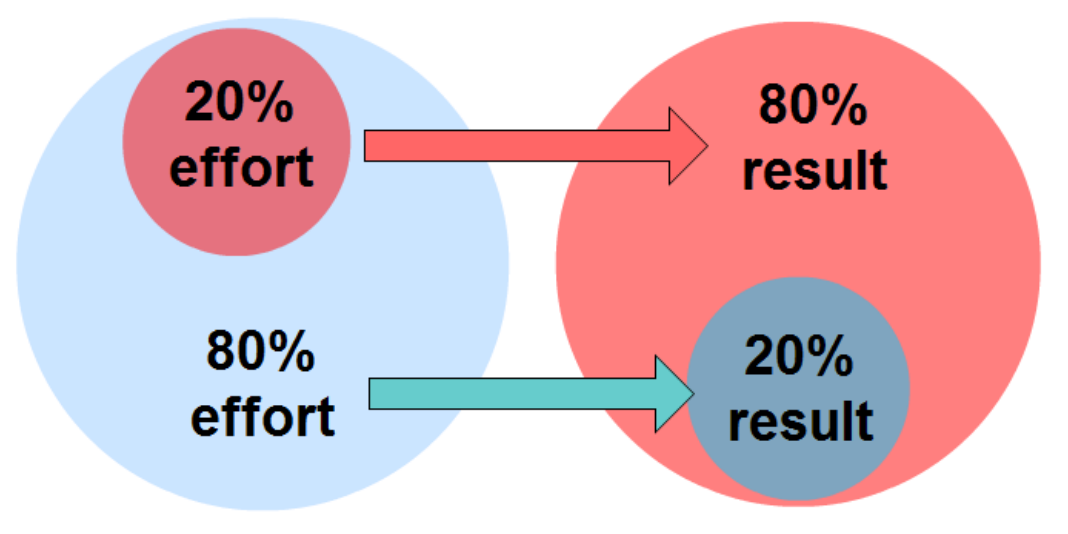

Figure 5: Pictorial Representation of the 80/20 Rule (http://www.comindwork.com/weekly/201509-14/productivity/pareto-principle-for-work-80-20-rule)

This rule can help managers identify problems and determine which operating factors (or people) are critical to the company's success. This is also referred to as the "Pareto Principle," "the principle of factor scarcity" and the "law of the vital few." Merely a few contributing factors, or select, productive people, are worth most.

Author Perry Marshall, believes that understanding the 80/20 rule can effectively increase your time management and productivity as an entrepreneur ${ }^{13}$. The United States would be back down to 5 percent unemployment if entrepreneurs stopped taking out their own $\operatorname{trash}^{13}$. Hiring employees to perform menial tasks (for a low wage), allows employers to improve their bottom line, and substantially increase their productivity ${ }^{13}$. If they remember the 80/20 Rule, that is, $80 \%$ of their business comes from $20 \%$ of their clients, they can further improve where they concentrate their efforts to increase their profits, see Figure 6.

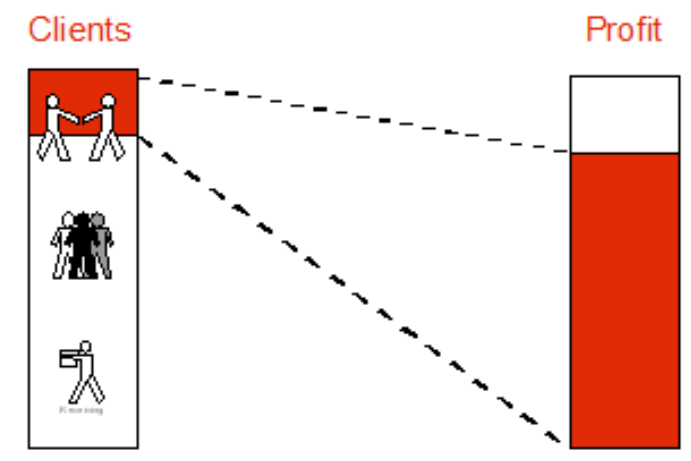

\section{The top $20 \%$ of your clients Generate $80 \%$ of your profit}

Figure 6: 80/20 Rule for Clients and Profit (https://www.pinterest.com/zd zoomdigital/growthstrategies/) 
Interestingly, this rule can also be applied directly to Software Development:

$80 \%$ of performance improvements are found by optimizing $20 \%$ of the code $20 \%$ of software bugs cause $80 \%$ of the software's failures ${ }^{14}$

Although many programmers, who spend hours on code, might seldom admit the truth out loud, somehow it works, without any verifiable reason. Thus, the more Rules of Thumb and 80/20 scenarios you are familiar with, the greater your advantage will be when structuring your company, and carrying out your work. Knowing how to estimate certain matters that tend towards a general solution can reduce stress and uncertainty if you know what to anticipate.

\section{Visual Method}

For physical/ visual matters, you can utilize the Visual Method and make an estimation based on appearance. Most people do not realize it but, they use visual estimations on daily basis, from determining whether they should take an umbrella that day, to the ripeness of their fruit, to picking which book to read. Whether we approve or not, everyone is guilty of "judging a book by its cover". How else would we make decisions? Throughout the day we are constantly in the process of binary decision making, saying 'yes' and 'no' as we please, based in part, on visual or sensory input cues. The majority of these decisions are made using our vision, or another one of our senses. For example, you judge food based on its smell and appears to guesstimate whether you want to eat it. However, at a restaurant you have to judge based on the description in the menu. Using this type of estimation, a person can make a split second decision with just a glance. Other times, you have to know where to look. For example, how do you know if an avocado is ripe? It is very difficult to tell at first glace. However, the trick is to check underneath the stem. If the stem is easily removed and the flesh is green underneath, your avocado is ripe ${ }^{15}$. Visual estimation makes it possible to estimate based on appearance. Interestingly this example can also be seen as a Rule of Thumb.

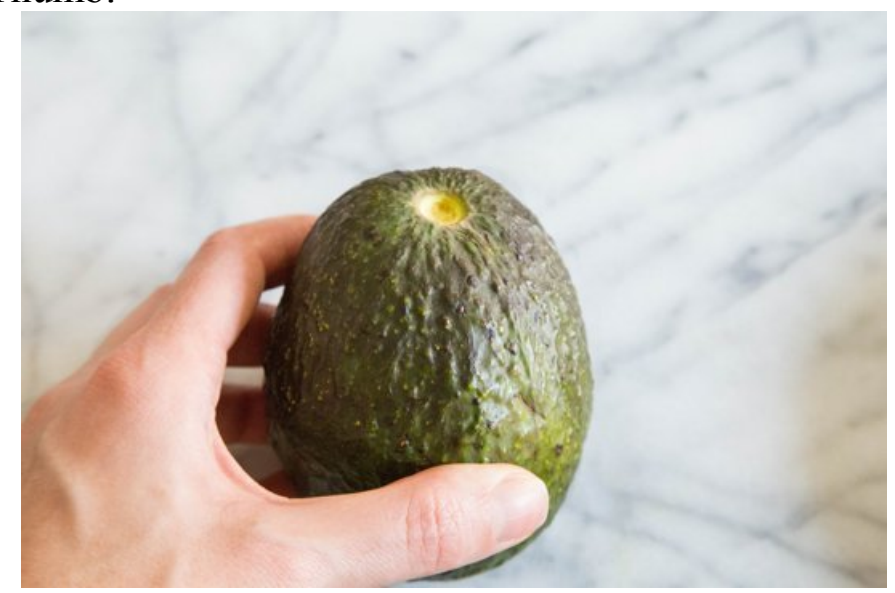

Figure 7: Checking whether or not an avocado is ripe (http://www.thekitchn.com/use-thissimple-trick-to-determine-whether-an-avocado-is-ripe-inside-tips-from-the-kitchn-172933)

Alike many of the other estimation methods, visual estimation is based on what you know or have experienced in the past. If you gaze at the moon, you can determine what day of the lunar 
month it is from its phase. A waxing crescent appears at the start of the lunar month, and a waning crescent appears at the end of the lunar month. If you know the stages in between you can guesstimate the date. Given a lunar cycle is approximately 28 days, if the moon is full, then it is about halfway through the month around the $14^{\text {th }}$ day. In other words, the new moon is at the beginning of the month, $1^{\text {st }}$ day, the waxing crescent phase between the $1^{\text {st }}-6^{\text {th }}$ days, $1^{\text {st }}$ quarter is after 1 week, Waxing Gibbous is between the $8^{\text {th }}-13^{\text {th }}$ days, Full Moon is the $14^{\text {th }}$ day, Waning Gibbons is $15^{\text {th }}-20^{\text {th }}$ days, Last Quarter is the $21^{\text {st }}$ day, and Waning Moon is between the $22-27^{\text {th }}$ days.

\section{Phases of the Moon}

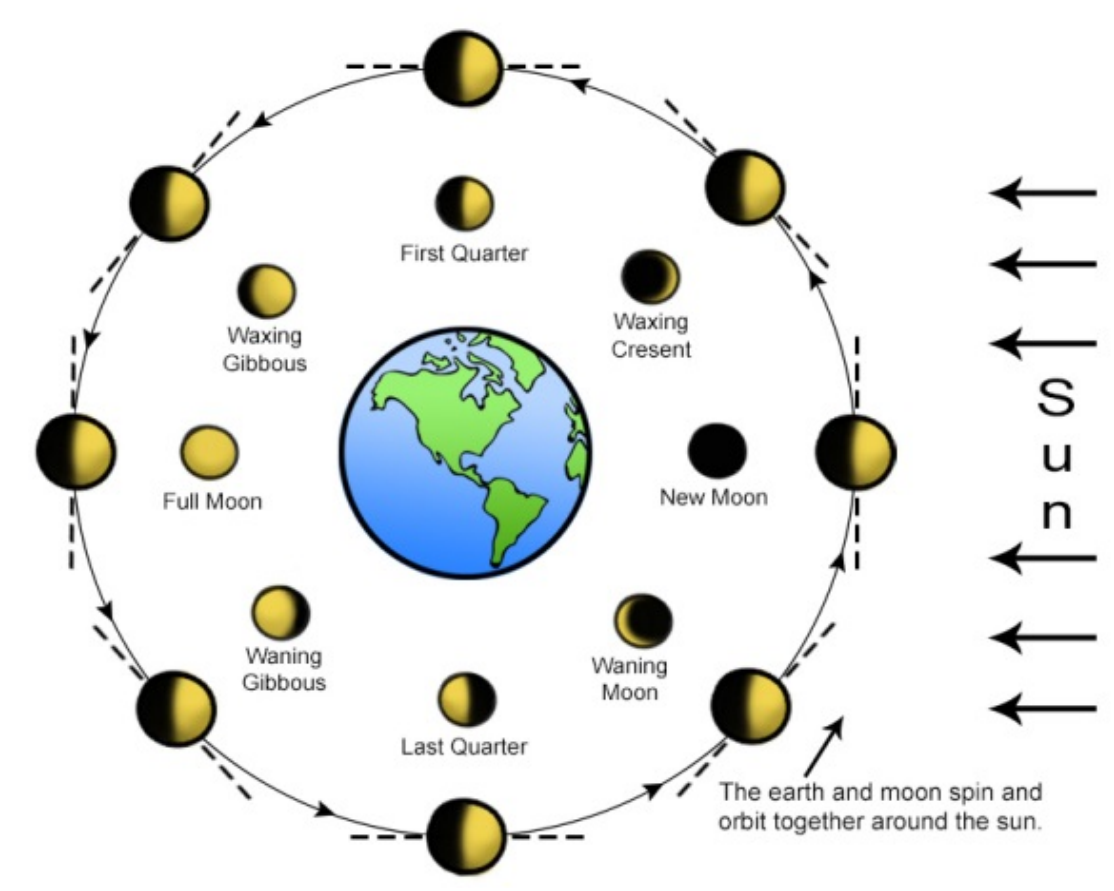

Figure 8: Phases of the Moon

\section{Upper and Lower Limits Method}

The Upper/Lower Limits method can also be applied to judge whether something is within bounds. This form of estimation is typically used in instances where you are only concerned that it falls within the maximum and minimum values.

In the case of manufacturing, quality control often comes is crucial. Quality control relies greatly on a set of statistics that address upper and lower control limits. While it has improved from the past, there are still some flaws. In order to catch these flaws limits are put in place to determine whether a product is effective. Some products need to be incredibly precise, whereas others need to be larger than one box, but smaller than another. For example, resistors can be made relatively cheaply, but companies acknowledge their consistency with a tolerance band. Some resistors have a tolerance of $10 \%$, others $5 \%$ or even smaller. This means that you can have a resistor 
marked $150 \Omega$, and its actual resistance will be $165 \Omega$ or $135 \Omega$. Depending on your project an estimate is an estimate, and the range is more important than the accuracy.

Unfortunately, estimation is only as good as you make it. Consider a time that you have been in the hospital and asked, "What is your pain level?" You have to estimate what your feeling would be if it were a number between 0 and 10. As in the Figure 9, you have to rate your experience within one of the listed categories based on the hints provided by the faces.

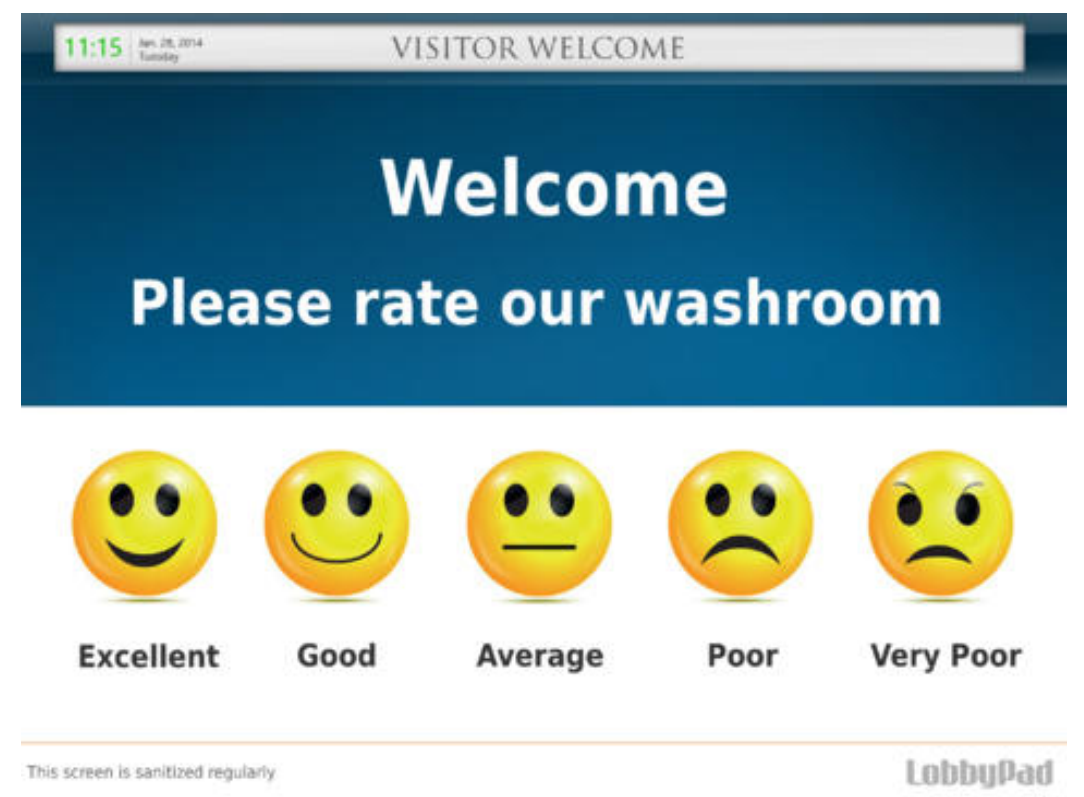

Figure 9: Visual Estimation Card (http://www.appster.org/app/ipad/lobbypad-smiley-facecustomer-feedback-843270731)

Similarly, precision is not necessary in all engineering applications; as long as you know that your estimate is within the range. Visual estimation is an important tool in pass fail testing, also known as Go/No-Go. Consider the snap gauge, in Figure 10.

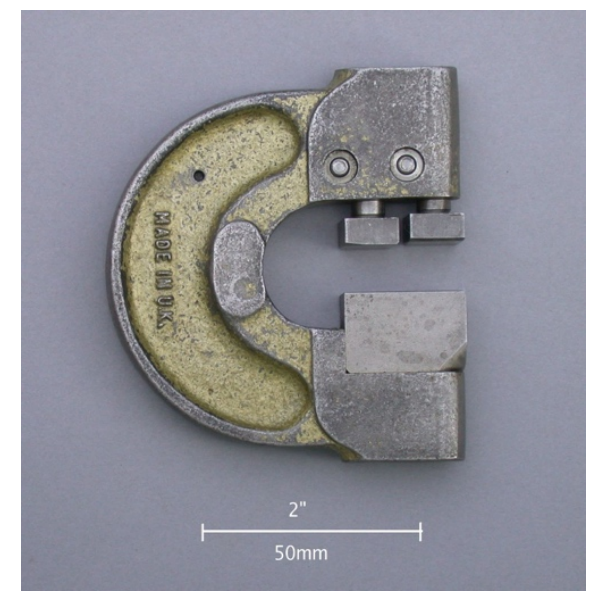

Figure 10 Snap Gauge

(https://en.wikipedia.org/wiki/Go/no_go_gauge\#/media/File:GaugeGapPlainGoNoGo.jpg) 
To determine whether a component is usable, it can be tested visually within the gauge. If the component is able to pass the first mechanical space, but not the second the component passes and is a "Go". If the component passes the first space and the second space in the gauge it fails and is a "No-Go". This method is much simpler than measuring each component with a Vernier caliper. In many cases the decimal places are irrelevant and merely time consuming. However, it is the engineer's job to determine not only when estimation is appropriate, but when it can make his/her job easier.

\section{Assessment}

The methods and examples highlighted in this paper were implemented in a Fundamentals of Engineering class taught at Florida Atlantic University. A questionnaire was conducted in order to gauge how receptive the students were to various learning techniques presented. The responses are based on an interactive class presentation that included the examples described in this paper. 62 students responded to the questionnaire. A summary of the survey responses can be found in the Appendix.

The answers of the questionnaire were based on a scale from 1-5, one being "strongly disagree," four being "strongly agree" and two being "neutral." Based on the in-class assessment survey, it was found that for:

Segmentation: 44\% Strongly Agree, 29\% Agree, 26\% Neutral

Fermi: 37\% Strongly Agree, 48\% Agree, 11.3\% Neutral

Rules of Thumb: 34\% Strongly Agree, 34\% Agree, 29\% Neutral

80/20 Rule: 39\% Strongly Agree, 39\% Agree, 21\% Neutral

Visual Methods: 39\% Strongly Agree, 34\% Agree, 23\% Neutral

Upper and Lower Limits: 42\% Strongly Agree, 37\% Agree, $13 \%$ Neutral

The results show that students are highly in favor of learning estimation methods, particularly in a visual and interactive environment. These findings are similar with those of Barry, Berry, Cunningham and Newton, who also studied applying estimation to engineering ${ }^{16}$.

\section{Conclusion}

Estimation is a fundamental tool that assists students in pursuing their entrepreneurial goals. Entrepreneurs are successful, in part, because they are visionary. They can visualize what the big picture looks like and they frequently rely on estimation to get there. An entrepreneur or an engineer, must always have a rough estimate for their cost, revenue, and time it takes to finish, on hand to make decisions. If they are pitching to an investor (shark), on the popular TV show "Shark Tank", those are some of the questions he/she is always asked". However, the sharks must also be heavily reliant on estimation. They must listen to a brief pitch and make a decision on whether or not they are going to invest in the entrepreneur's product. They might estimate whether the product makes sense to add to their brand, how long it will take to recoup their 
money, and the commercialization of the product. Early insight and quick calculation can be incredibly helpful.

By keeping this in mind, students can benefit greatly from the addition of simple estimation methods to their curriculum. Student responses indicated their preference to learn these methods were heightened by visually engaging material and multiple examples to enhance their understanding of the ways the methods can be used to solve problems and think creatively. In order to make a "good estimation", it is necessary to understand why you are using it.

Although some skeptics will continue to ask the question, "why do we even need estimation when nowadays, we can write programs to calculate nearly anything?". In some instances, usually when working in teams, estimating is about creating a shared understanding of a given requirement, and keeping the big picture in mind. By keeping things simple, and not getting caught up in precise details of the final results; estimation puts everyone on the same page to work toward a common goal. Although the estimates will not always be exactly right, they will be consistent.

\section{Future Work}

This paper is part of an ongoing work that includes additional estimation techniques and examples that are relevant to engineers and entrepreneurship. Some of the additional estimation techniques we are delving into include: accuracy vs. precision, fuzzy logic, common sense, binary decision making, and gut feeling. We are also working on estimation teaching methods, with an increased number of engineering applications, and the use of puzzles and visual methods to explain these topics.

\section{Acknowledgements}

We thank the Venturewell.org (formerly NCIIA.org), for the support of the development of innovative and entrepreneurial teaching and learning methods. We also thank Michael R. Levine and Last Best Chance, LLC, for the continuous support.

We thank Deana Karp for her earlier contributions to the paper.

\section{References}

[1] S. Shakerin, "The Art of Estimation", International Journal of Engineering Education, vol. 22, no. 2, 2006.

[2] B. Linder, Understanding estimation and its relation to engineering education, 1 st ed. Boston: Massachusetts Institute of Technology, 1999. 
[3] R. Bourn and S. Baxter, "Developing Mathematical Intuition by Building Estimation Skills", in 2013 ASEE Annual Conference, Atlanta, 2013.

[4] A. Lehrman and H. Nachtmann, "Teaching Engineers To Handle Uncertainty", in 2002 Annual Conference, Montreal, 2016.

[5] S. Murphy and S. Schindler, Betcha!. New York, NY: HarperCollins Publishers, 1997.

[6] B. Goldstone, Great estimations. New York: Henry Holt and Co., 2006.

[7] B. Goldstone, Greater estimations. New York, N.Y.: Henry Holt and Co., 2008.

[8] A. Santos, How many licks?. Philadelphia: Running Press, 2009.

[9] Rainbow.ldeo.columbia.edu, "Fermi's Earth Circumference", 2016. [Online]. Available: http://rainbow.ldeo.columbia.edu/courses/v1001/fermi.html.

[10] L. Weinstein, Guesstimation 2.0. Princeton, N.J.: Princeton University Press, 2012.

[11] J. Welsh, "Gimli Glider' Pilot Recalls Landing Without Engines 30 Years Ago", Wall Street Journal, 2013.

[12] T. Parker, Never trust a calm dog, and other rules of thumb. New York, NY: HarperPerennial, 1990.

[13] P. Marshall, "The 80/20 Rule of Time Management: Stop Wasting Your Time", Entrepreneur, 2013. [Online]. Available: http://www.entrepreneur.com/article/229813.

[14] WhatIs.com, "What is Pareto principle? - Definition from WhatIs.com", 2016. [Online]. Available: http://whatis.techtarget.com/definition/Pareto-principle.

[15] D. Velden, "Use This Simple Trick to Determine Whether an Avocado Is Ripe Inside Tips from The Kitchn", The Kitchn, 2015. [Online]. Available: http://www.thekitchn.com/usethis-simple-trick-to-determine-whether-an-avocado-is-ripe-inside-tips-from-the-kitchn-172933.

[16]A. Barry , D. Berry, S. Cunningham, J. Newton, M. Schweppe, A. Spalter, W. Whitely, R. Williams, "Visual Learning for Science and Engineering," A Visual Learning Campfire, June 2014.

[17] ABC, "About Shark Tank TV Show Series", 2016. [Online]. Available: http://abc.go.com/shows/shark-tank/about-the-show. 


\section{Appendix: Student Responses to Estimation Methods}

The answers of the questionnaire were based on a scale from 1-5, one being "strongly disagree," four being "strongly agree" and two being "neutral." Based on the in-class assessment survey, it was found that:
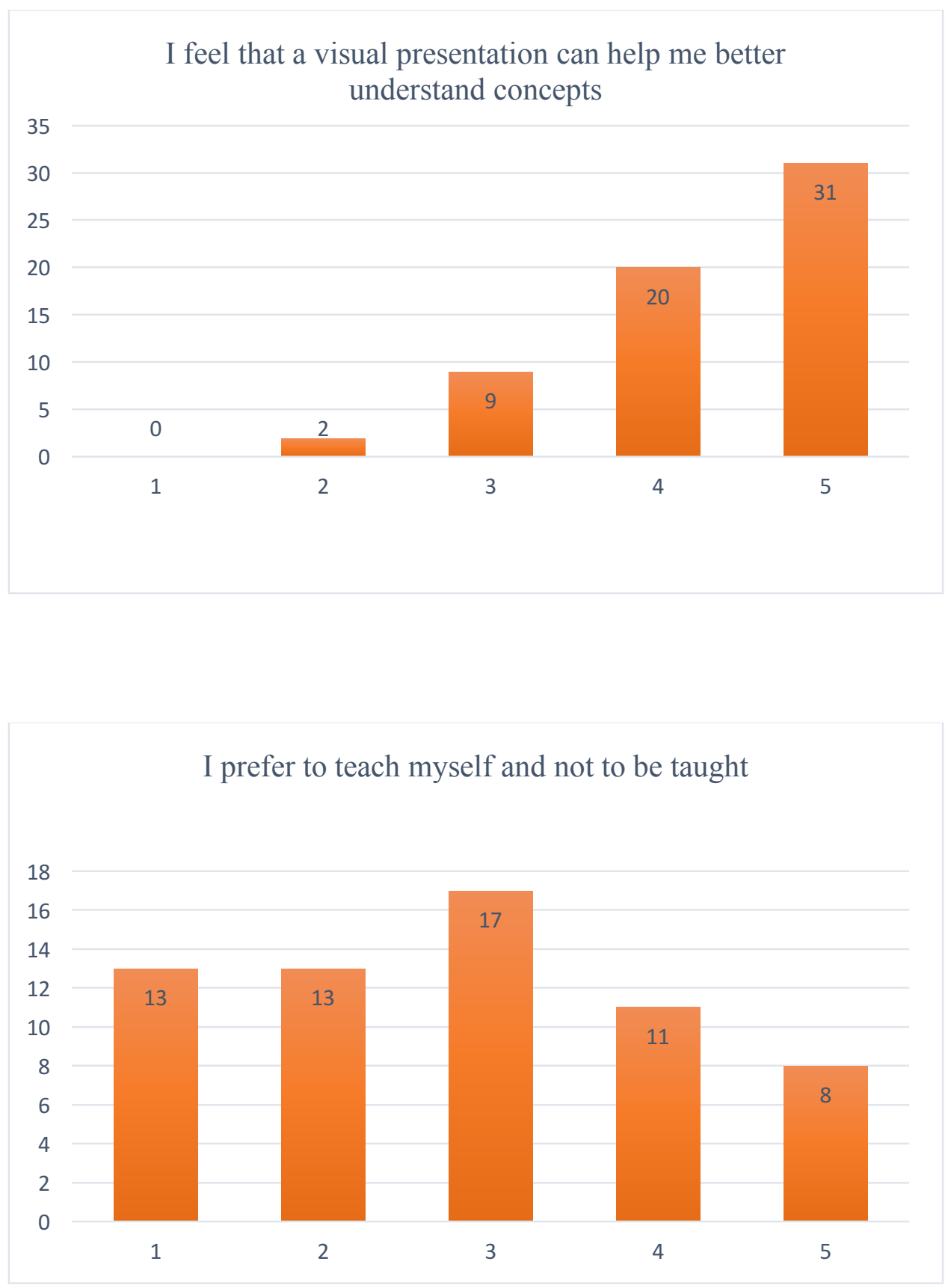


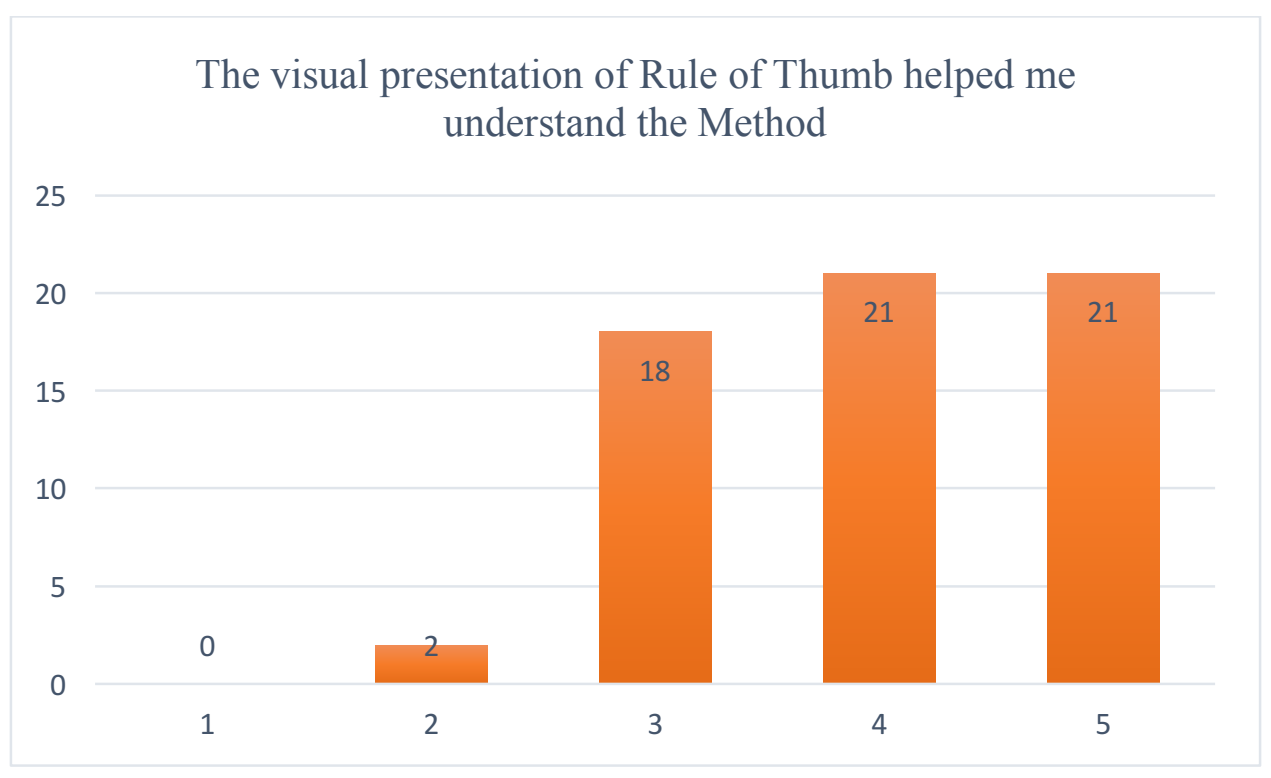

This visual presentation helped me better understand the 80/20 Method

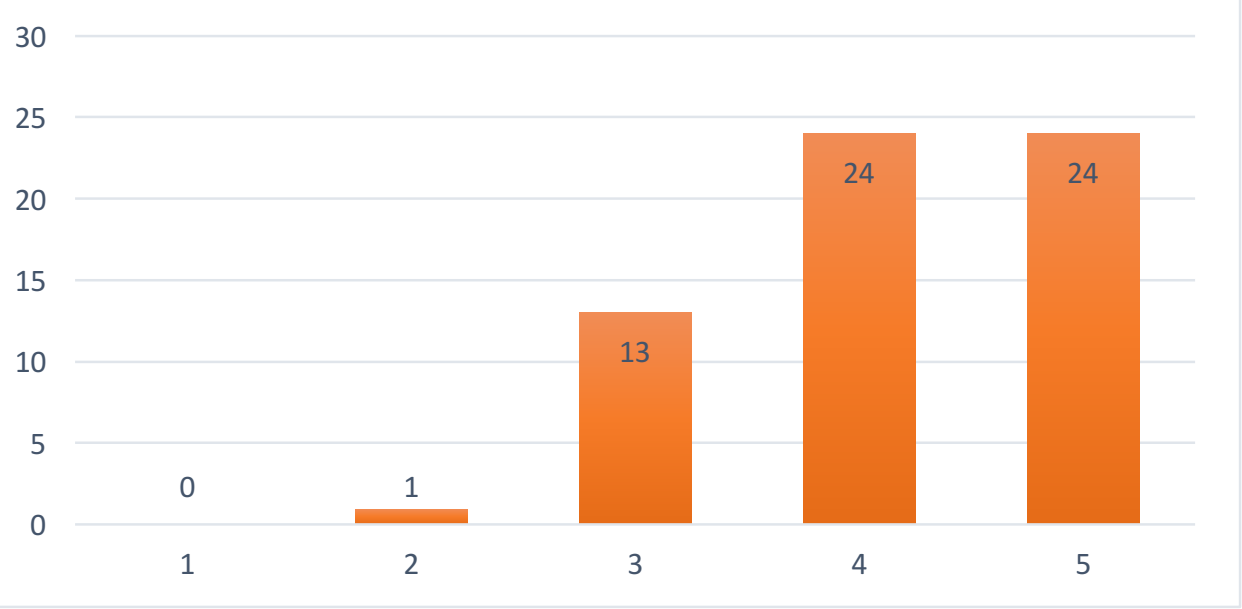



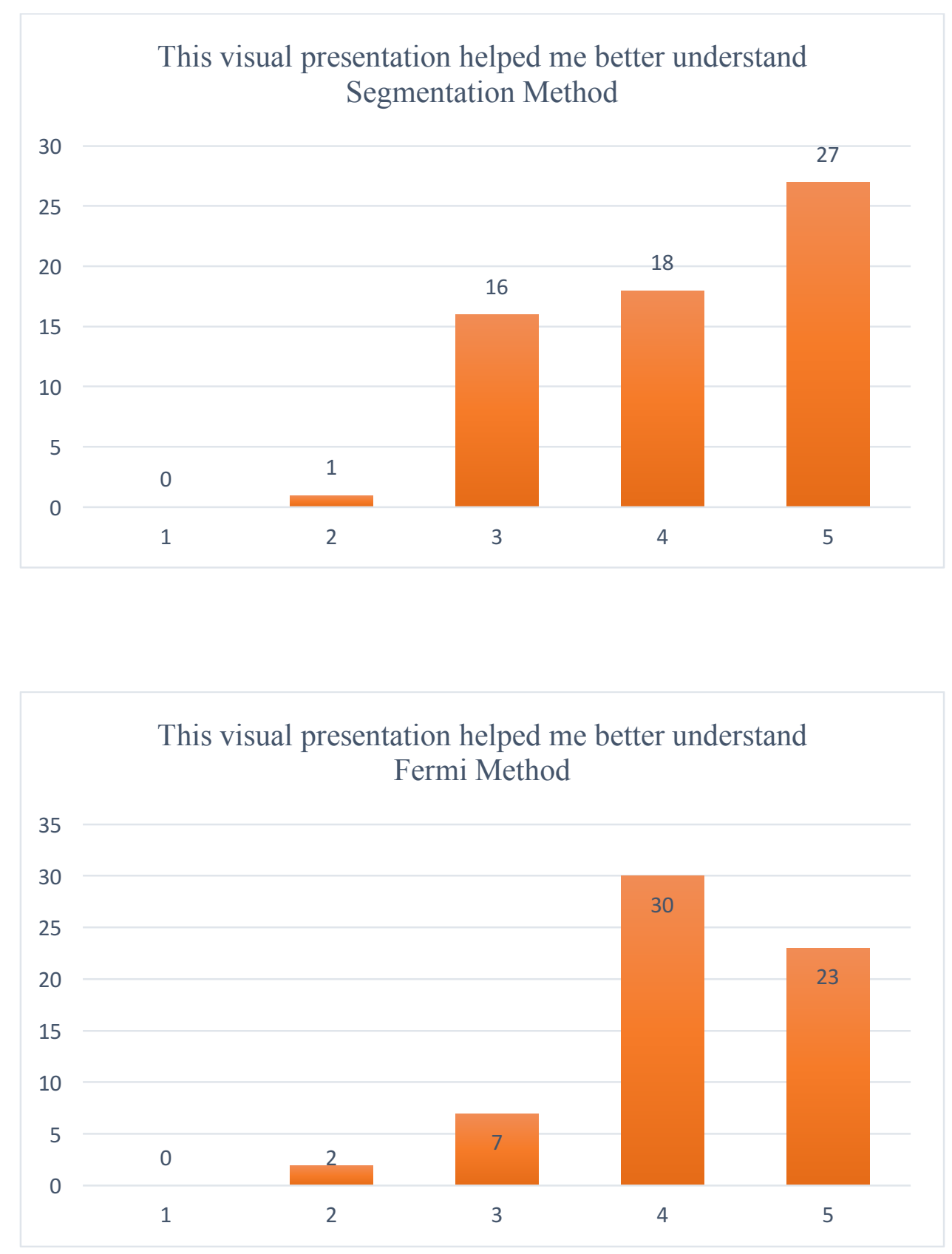

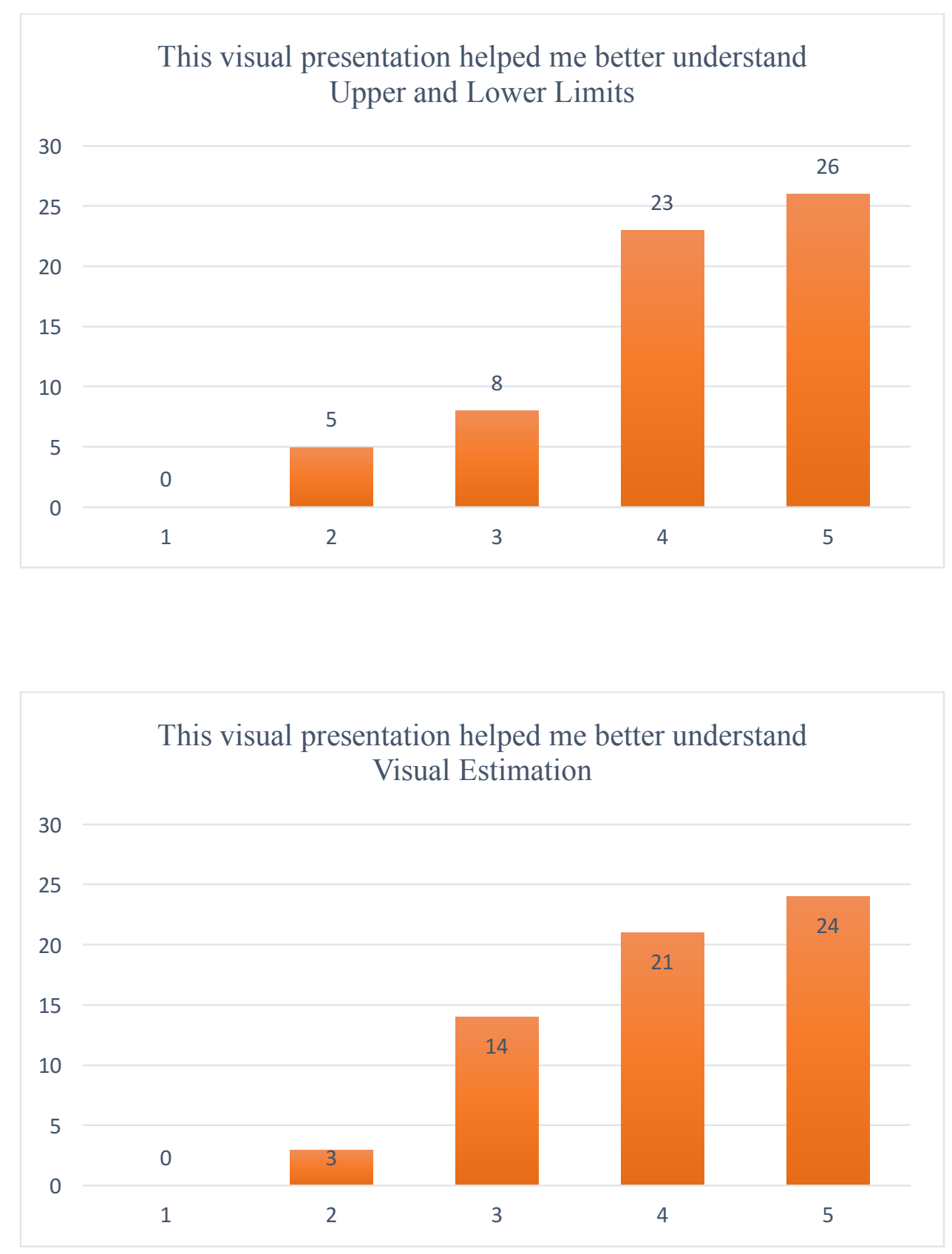


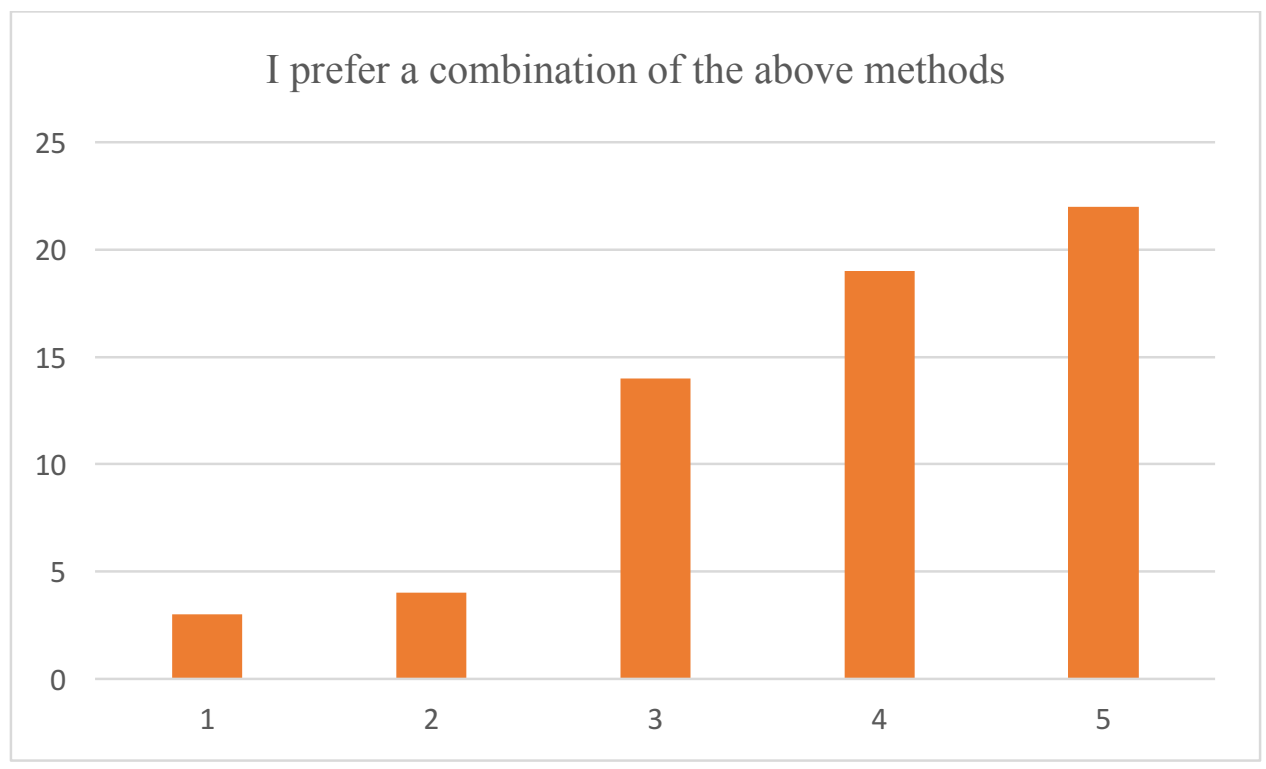

